\title{
A Double Take on Unconventional Photon Blockade
}

\section{Two separate groups have demonstrated a photon blockade effect that could be used to make practical single-photon emitters.}

\section{by Marina Radulaski*}

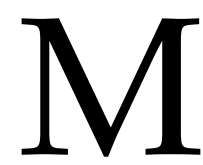

any quantum technologies require light sources that emit a precise number of photons. Examples include quantum cryptography, which uses individual photons for secure communication, and quantum imaging, which harnesses wave packets of a fixed number of photons to achieve enhanced resolution. A promising method for quickly generating single photons exploits an emitter-cavity system and the so-called photonblockade effect. Here, the system acts as a photon filter for a laser, allowing the transit of only one photon at a time. Now two independent teams have overcome a major engineering challenge to realizing photon blockade in practical systems $[1,2]$. These demonstrations take the effect one step closer to implementation in quantum technologies.

A typical photon-blockade device for optical photons consists of a cavity containing an emitter with two energy levels, such as an atom or a quantum dot. The presence of the emitter alters the cavity's properties in such a way that the cavity can only accommodate a single photon. Consequently, if a pulse of light from a laser is introduced into one end of the cavity, then the other end will only emit one photon. This blocking effect is a bit like a single-lane exit on a multilane highway, which only allows cars to leave the highway oneby-one. Photon blockade was demonstrated in the lab [3, 4] more than a decade ago. But realizing the effect with high photon emission rates required a strong interaction between the emitter and cavity, which entailed challenging, high-precision fabrication of cavities.

An alternative route to achieving photon blockade arose in 2010, when theorists predicted that the strong interaction restriction could be relaxed if two cavities were used instead of one [5]. These two coupled cavities would require only weak interactions with the emitter to generate single photons [6], and, as such, they would not need to be as precisely constructed. This alternative approach relies on what is known as the unconventional photon blockade effect. To appreci*Edward L. Ginzton Laboratory, Stanford University, Stanford, CA
94305, USA

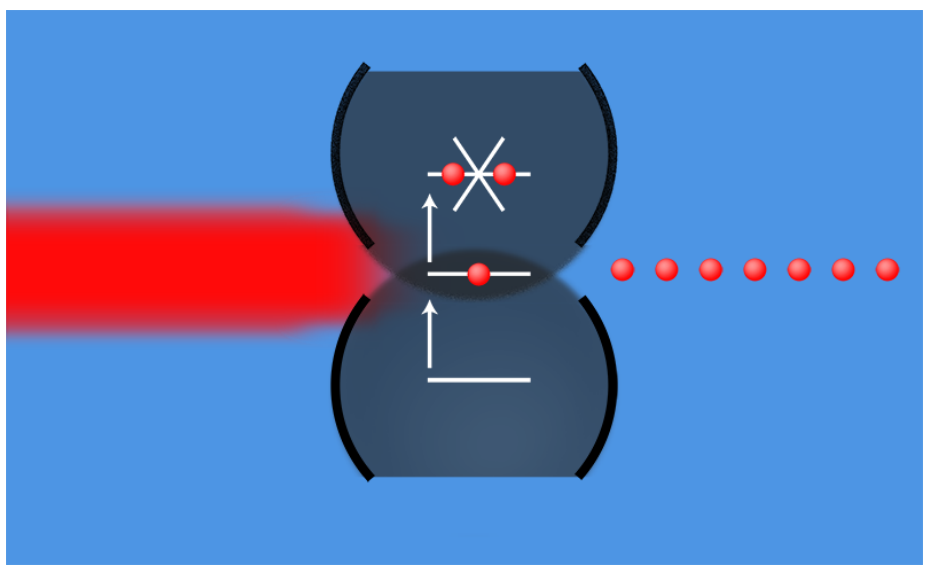

Figure 1: A scheme for single-photon emission using the unconventional photon blockade effect. The device consists of two coupled cavities, which are weakly coupled to a dipole emitter (not shown). Population of the system by two photons is blocked via destructive interference of the different excitation paths. (APS/Joan Tykco)

ate its distinction from "regular" photon blockade requires some details about how photon blocking works. Cavities have a series of equally spaced energy levels, $N=1,2,3, \ldots$, which correspond to $1,2,3, \ldots$, photons occupying the cavity. For regular photon blockade, the presence of an emitter shifts all the energy levels of the cavity such that only the $N=1$ energy level can accept photons. Photons tuned to this energy can therefore only go in and out of the cavity one at a time. Unconventional photon blockade is less restrictive: it blocks the entry of two photons only. This blocking is achieved via destructive interference of disparate excitation paths to the $N=2$ energy level (Fig. 1). But the effect does not influence the $N=3$ and higher energy levels in the same way, allowing emission of "bunches" of three or more photons.

Attempts to realize unconventional photon blockade have been hindered by a lack of suitable platforms. But a recent theoretical paper [7] proposed several new experimental approaches. Inspired by one idea for optical photons, Henk Snijders of Leiden University, Netherlands, and collabora- 
tors have now realized unconventional photon blockade using a quantum dot and an optical cavity that functions like two cavities [1]. Specifically, the team used two orthogonally polarized modes of the same cavity. Each mode was weakly and unequally coupled to the emitter. The team showed that when both modes were excited equally, the system emitted single photons as predicted for the unconventional photon blockade effect. They also showed that their photon emission rate was 10 times higher than that achieved in other schemes for generating single photons using weakly coupled systems.

Complementary experiments by Cyril Vaneph and collaborators at the University of Paris-Sud [2] achieved unconventional photon blockade with microwaves. Their setup consisted of two coupled superconducting niobium cavities. One cavity contained a superconducting quantum interference device, or SQUID, which altered the cavity's response to incoming microwaves and blocked the transmission of two microwave photons.

The two experiments introduce novel ways of achieving photon blockade effects. But the new cavity systems are still some way from being practical for quantum technologies. For example, the devices aren't perfect blockers: they can occasionally emit two photons or photon bunches containing three or more photons. Further study is needed to see whether the photon-bunch emission of these devices can be experimentally controlled, for example, by using the intensity of the input laser or microwaves. Researchers may find that there is an inherent tradeoff between the single-photon emission rate and contamination of the singlephoton source with photon bunches. Another intriguing behavior observed in the microwave experiment is photon emission oscillation, where the device rapidly switches between single-photon and photon-bunch emission. Whether such oscillations would be an opportunity or an impediment for quantum protocols is, as yet, unexplored.

Compared with existing single-photon emitters, the new devices developed by Snijders et al. and by Vaneph et al. will likely be easier to integrate into quantum circuits. Such an advance may nudge quantum technologies closer to practical use. Personally, I am looking forward to the realization of unconventional photon blockade in other optical systems, such as those based on cavities hosting crystal defects called color centers [8]. Compared to quantum dots, color centers have much more homogeneous properties, and their host devices should emit identically-colored single photons, opening up opportunities in terms of scalability. Finally, I am excited to see if the approach from Vaneph and collaborators can be used to generate single microwave photons in superconducting cavity arrays [9]. Such arrays could provide the hardware for quantum simulation of many interesting and complex systems.

This research is published in Physical Review Letters.

\section{REFERENCES}

[1] H. J. Snijders, J. A. Frey, J. Norman, H. Flayac, V. Savona, A. C. Gossard, J. E. Bowers, M. P. van Exter, D. Bouwmeester, and W. Löffler, "Observation of the Unconventional Photon Blockade," Phys. Rev. Lett. 121, 043601 (2018).

[2] C. Vaneph, A. Morvan, G. Aiello, M. Fechant, M. Aprili, J. Gabelli, and J. Esteve, "Observation of the Unconventional Photon Blockade in the Microwave Domain," Phys. Rev. Lett. 121, 043602 (2018).

[3] K. M. Birnbaum, A. Boca, R. Miller, A. D. Boozer, T. E. Northup, and H. J. Kimble, "Photon Blockade in an Optical Cavity with One Trapped Atom," Nature 436, 87 (2005).

[4] A. Faraon, I. Fushman, D. Englund, N. Stoltz, P. Petroff, and J. Vučković, "Coherent Generation of Non-Classical Light on a Chip via Photon-Induced Tunneling and Blockade," Nat. Phys. 4, 859 (2008).

[5] T. C. H. Liew and V. Savona, "Single Photons from Coupled Quantum Modes," Phys. Rev. Lett. 104, 183601 (2010).

[6] M. Bamba, A. Imamoğlu, I. Carusotto, and C. Ciuti, "Origin of Strong Photon Antibunching in Weakly Nonlinear Photonic Molecules," Phys. Rev. A 83, 021802 (2011).

[7] H. Flayac and V. Savona, "Unconventional Photon Blockade," Phys. Rev. A 96, 053810 (2017).

[8] M. Radulaski, K. A. Fischer, K. G. Lagoudakis, J. L. Zhang, and J. Vučković, "Photon Blockade in Two-Emitter-Cavity Systems," Phys. Rev. A 96, 011801 (2017).

[9] C. Eichler, Y. Salathe, J. Mlynek, S. Schmidt, and A. Wallraff, "Quantum-Limited Amplification and Entanglement in Coupled Nonlinear Resonators," Phys. Rev. Lett. 113, 110502 (2014).

10.1103/Physics. 11.74 